\title{
Determinants and Heterogeneity of Time -to- Recovery from Obstetric Fistula Patients; Comparison of Acceleration Failure Time and Parametric Shared Frailty Models
}

\author{
Million Wesenu Demissie ${ }^{1}$ \\ ${ }^{1}$ Department of Statistics, College of Computing and Informatics, Haramaya University, Ethiopia \\ Correspondence: Million Wesenu Demissie, Department of Statistics, College of Computing and Informatics, Haramaya \\ University, Dire Dawa, Ethiopia
}

Received: October 30, 2017

Accepted: November 22, 2017 Online Published: December 13, 2017

doi:10.5539/ijsp.v7n1p99

URL: https://doi.org/10.5539/ijsp.v7n1p99

\begin{abstract}
Obstetric Fistula is a medical condition that involves an opening or perforation between the vagina and the bladder or the vagina and the rectum. It is a serious, life threatening and often debilitating medical condition that affects thousands of women in developing countries.The study consists of 270 obstetric fistula patients having all required information who were taking treatment at Jimma University Specialized Hospital in south west Ethiopia from January, 2011 to January ,2017. The log-rank and generalized wilcoxon test were used to explore the association between the recovery time and different independent categorical covariates. Then using different baseline distribution parametric models were employed to have an appropriate model for the recovery time/status of the patients based on Akaike information criteria (AIC) of the model. Result of Both log-rank and generalized wilcoxon test showed that there were significant differences among obstetric fistula patients in survival experience of weight , marital status, Residence, Incontinence, Antenatal care, mode of delivery, status of urethra and types of fistula of patients at $5 \%$ level of significance. Based on AIC log-normal gamma shared frailty model is an appropriate model and there is heterogeneity between patients with zone. The final model showed that marital status, Residence, Incontinence, Antenatal care, status of urethra and types of fistula were the determinants of recovery status of the patients at $5 \%$ level of significance. In Conclusion, the result showed that married women, rural residence, incontinence of urine more than 3 months, hadn't antenatal care and completely damaged urethra were prolonged time recovery time of patients whereas having recto-vaginal-fistula shorten recovery status than vesico-vaginal fistula group of patients.
\end{abstract}

Keywords: acceleration failure time, frailty, gamma shared frailty, obstetric fistula, recovery

\section{Introduction}

Obstetric fistula is one of the most devastating medical disabilities afflicting women as a result of complications arising from lack of surgical intervention for prolonged labor and is a serious, life threatening and often debilitating medical condition that affects thousands of women in the world. An estimated 2-3 million women globally and majorly in sub-Saharan Africa and Asia, suffer from fistula with an annual incidence of 50,000-100,000 women (Wall et al.,2005 \& Tuncalp et al.,2015).Obstetric Fistula (OF) is a medical condition that involves an opening or perforation between the vagina and the bladder or the vagina and the rectum. Though global in its prevalence it is very rare in the developed world and its cause is mainly from malignant diseases, radiation therapy, or surgical malfunctions during delivery (Goodwin \& Scardino, 1980, Langkilde et al.,1999).

In the developing world, Obstetric Fistula is a gynecological complication leading to urinary/faecal incontinence resulting mainly from prolonged obstructed labor. Even though there might be minor inter-country differences, the complication is mainly due to adolescent pregnancy from early marriage exacerbated by lack of access to emergency obstetric care (Muleta et al., 2008). The higher rates of fistula in many developing countries (as compared to industrialized countries) are not only due to poverty, but also due to the impact of come cultural practices on women's status, health and wellbeing. One of the harmful cultural practices seen in some communities is the act of giving women lots of water to drink during labor; a full bladder has a higher tendency to cause fistula during expulsion of the baby (Cook et al., 2004)

Obstructed labor does not happen in isolation. There are many factors that could lead up to this unfortunate situation. In Tanzania, Kazaura et al., (2011) conducted a cross sectional survey to determine the awareness and perceived causes of OBF in a rural area of Tanzania. They found that among 334 OBF patients, $72.2 \%$ were within the $25-29$ years age group. 
Of the 291 mothers who reported to have delivered their youngest child, about two thirds $65 \%$ reported to have had their babies at a health facility. Lack of money, lack of family support and long distance to health facilities were some of the reason that made women delivery at other places than health facilities. Holme et al., 2007 conducted a retrospective cross-sectional study at a hospital in Lusaka, Zambia. Of the 259 women with OBF, one-quarter were below age 20 years. About $75 \%$ of the women who had fistula were married, $15.1 \%$ were divorced while $1.7 \%$ were single. The study further showed that most women who suffer from fistula have a small and short stature. It was also found that, women lose weight when they developed fistula which may be due to the neglect they receive for having the condition.

OBF remains a constant cause of misery to women of childbearing age because of the continuous leaking of urine or feces, and the constant wetting of their clothes coupled with the offensive smell produced. Some communities even consider them as outcasts and OBF victims are sent out of their villages because community members think that the condition is contagious. Even when the fistula itself has been repaired, some women still go through other ordeals such as urinary (stress) incontinence, vaginal stenosis or even atresia and infertility. Obstetric fistula is a physically and socially disabling obstetric complication that affects many women annually. It has a devastating social, economic and psychological effect on the health and well-being of the affected women (Mselle et al., 2011). The stigma, deep sense of loss and loss of dignity and identity associated with fistula has a negative impact on quality of life (Kirby et al., 2014).

In addition to the psychological trauma inflicted, obstetric fistula also imposes enormous medical / financial burdens on victims and their families. Because of the stigma attached to the condition, people suffering from the disease are also not very easy to locate. The study conducted in eastern Ethiopia at Harar Hamlin Fistula Center showed that mean survival time of obstetric fistula patients to become recovered was 18.71 days with standard deviation of 6.68 days and having complete bladder neck distraction (AHR=0.1324, CI: 0.0360, 0.4867), partial urethral damage (AHR=0.6929, CI: 0.4812 , 0.9976 ) and severe vaginal scaring (AHR=0.269, CI: $0.1399,0.5174$ ) have significant effect on mean time to recovery from obstetric fistula Cox proportional hazard analysis (Hussen \& Melese, 2017).

This Study was intended to model survival time-to-Recovery from Obstetric Fistula in southwest, Ethiopia using survival analysis framework. The primary variable in survival analysis is survival time, time-to-Recovery from Obstetric Fistula for this study. It was investigated the major risk factors associated to recovery of women from Fistula which will help to guide health professionals and health policy makers to identify indicators for monitoring women with obstetric fistula and applying necessary preventive and appropriate measures to decrease time to recovery of patients and may ultimately it will helps to reduce attitude of community members those consider fistula as contagious.

The survival approach used for this, were non-parametric, semi-parametric and parametric with (weibull, log-logistic and log normal distribution) using shared frailty model having frailty distribution of gamma and inverse Gaussian. Frailty models account for unobserved heterogeneity that occurs because of some observations are more prone to failure and therefore frail than others in a data set. Therefore, the objective is to introduce an additional parameter to the hazard rate that accounts for the random frailties. These frailties can be specific to individuals or groups, and are referred to as individual frailty or shared frailty respectively. The shared frailty model is relevant to event times of related individuals, similar organs and repeated measurements. Individuals in a cluster are assumed to share the same frailty, which is why this model is called shared frailty model. It was introduced by (Clayton, 1978)_and extensively studied in (Hougaard, 2000). Therefore, in this study, there may be similar frailty with in subcategories of fistula patients, based on living geographical area of women specifically zone.And the model best fit the data were selected using Akaike information criteria (AIC) and Bayesian information criteria(BIC).

\section{Methods and Material}

\subsection{Source of Data}

All medical records of obstetric fistula patients those who were registered to fistula ward at Jimma University Specialized Hospital from January, 2011 to January, 2017 in south west Ethiopia were retrospectively reviewed by medical professionals. The data consists of women that admitted to Hospital with obstetric fistula case. The total numbers of women registered during this study were 292. Among the total of 292 of women registered in the given year, only 270 women with obstetric fistula whose card had full information and hence are included in this study.Data analysis was performed using STATA software.

\subsection{Variables in the Study}

The response (dependent) variable is continuous and describes the length of hospital stay time in days. The response variable for the $i^{\text {th }}$ individual is represented by $Y_{i}$ and it measures duration to event and it is defined by status variable (event or censoring variable). Survival time measures the follow-up of time from a defined starting point to the occurrence of a given event. This observation time has two components, the beginning point of the study time and the observation of time to the end. In survival analysis, the outcome of interest (recovery in this study) is the duration of time until physically 
cured measured in weeks.

Table 1. Description of Covariates those were included in the study.

\begin{tabular}{|c|c|}
\hline Covariates & Categories \\
\hline 1. Age of patients & $0=<20, \quad 1=20-30 \quad$ and $2=>30$ \\
\hline 2. Weight of patient & $0=\leq 50 \mathrm{~kg} \quad, 1=>50 \mathrm{~kg}$ \\
\hline 3. Marital status & $0=$ others, $1=$ married, $2=$ divorced \\
\hline 4. Parity & $0=$ one child , $1=2-4$ children, $2=\geq 5$ children \\
\hline 5. Residence & $0=$ urban, $1=$ rural \\
\hline 6. Educational status & $0=$ illiterate $\quad, 1=$ literate \\
\hline 7. Incontinence of urine & $0=>3$ months, $1=\leq 3$ months \\
\hline 8. Antenatal care & $0=$ yes, $1=$ no \\
\hline 9. Duration of labor & $0=\leq 2$ days $\quad, 1=>2$ days \\
\hline 10. Place of residence & $0=$ health institution, $1=$ home \\
\hline 11. Mode of delivery & $0=$ spontaneous vertex $($ vaginal $), 1=$ cesearem section $(\mathrm{cs})$ \\
\hline 12. Surgery approach & $0=$ vaginal, Abdominal \\
\hline 13. Duration of catheter & $0=\leq 14$ days,$\quad 1=21$ days \\
\hline 14. Status of urethra & $0=$ partially damaged, $1=$ completely damaged \\
\hline 15. Types of fistula &, $1=\mathrm{RVF}$ \\
\hline
\end{tabular}

Note. $\mathrm{VVF}=$ vesico-vaginal fistula, $\mathrm{RVF}=$ recto vaginal fistula

\section{Method of Data Analysis}

\subsection{Non-parametric Survival Analysis}

The Kaplan-Meier (KM) estimator is the standard non-parametric estimator of the survival functions $(t)$, proposed by Kaplan and Meier (1958), and is also called the Product-Limit estimator. KM estimator incorporates information from all of the observations available, both censored and uncensored, by considering any point in time as a series of steps defined by the observed survival and censored times. When there is no censoring, the estimator is simply the sample proportion of observations with event times greater than $\mathrm{t}$. suppose $t_{1}, t_{2}, \ldots t_{n}$ be the survival times of $n$ independent observations and $t_{1} \leq t_{2} \leq \cdots t_{m}, m \leq n$ be the $m$ distinct ordered birth times. The Kaplan-Meier estimator of the survivorship function (or survival probability) at timet, $s(t)=P(T>t$ ) is defined as:

$$
\widehat{s}(t)=\prod_{t(j) \leq t}\left(\frac{n j-r j}{n j}\right)=\prod_{t(j) \leq t}\left(1-\frac{r j}{n j}\right)
$$

With the convention that, $\hat{S}(t)$ fort $<t_{(1)}$. Where $n_{j}$ is the number of individuals who are at risk at time $t_{j}$ and $r_{j}$ is the number of individuals who occurs an event at time $t_{j}$.

\subsubsection{Non Parametric Comparison of Survival Functions}

Log-rank test: Among the various non-parametric tests one can find in the statistical literature, the Mantel-Haenzel test, 
currently called the "log-rank" is the one commonly used non-parametric tests for comparison of two or more survival distributions. The log rank test statistic for comparing two groups is given by (Cox, 1984). The general form of this statistic is given by:

$$
\mathrm{Q}=\frac{\left[\sum_{i=1}^{m} w_{i}\left(d_{1 i}-\hat{e}_{1 i}\right)\right]^{2}}{\sum_{i=1}^{m} w_{i}^{2} \hat{v}_{1 i}} \sim \chi_{k-1}^{2}
$$

Where $\hat{e}=\frac{n_{1 i} d_{i}}{n_{i}}$ and $\hat{v}=\frac{n_{1 i} n_{0 i} d_{i}\left(n_{1 i}-d_{i}\right)}{n_{i}^{2}\left(n_{i}-1\right)}$, n0i and $\mathrm{n} 1 \mathrm{i}$ are the number at risk at observed survival time $\mathrm{t}(\mathrm{i})$ in group 0 and 1 respectively. $\mathrm{ni}$ is the total number of individuals or risk before time $\mathrm{t}(\mathrm{i}), \mathrm{d} 1 \mathrm{i}$ is the number of observed event in group 1 and di is the total number of event at $\mathrm{t}(\mathrm{i})$. also $\mathrm{k}$ is number of groups in each category. If weights (wi) equal to 1 , $\mathrm{i} . e$. wi $=$ 1, the Log Rank test (QLR) is used; otherwise if wi = ni, the Generalized Wilcoxon test (QGWt) is used.

$$
\mathrm{Q}_{\mathrm{LR}}=\frac{\left[\sum_{i=1}^{m}\left(d_{1 i}-\hat{e}_{1 i}\right)\right]^{2}}{\sum_{i=1}^{m} \hat{v}_{1 i}} \text { and } \mathrm{Q}_{\mathrm{GWt}}=\frac{\left[\sum_{i=1}^{m} n_{i}\left(d_{1 i}-\hat{e}_{1 i}\right)\right]^{2}}{\sum_{i=1}^{m} n_{i}^{2} \hat{v}_{1 i}}
$$

\subsection{Cox Proportional Hazard Model}

The Cox proportional hazards model is a semi- parametric model for fitting survival data which describes the relation between the event incidence, as expressed by the hazard function and covariates that influence survival time. Let $t$ denote a continuous non-negative random variable representing survival time. The basic Cox model is written as,

$$
h(t)=h_{o}(t) \exp \left\{\beta_{1} z_{1}+\beta_{2} z_{2}+\cdots \beta_{p} z_{p}\right\}
$$

Where $h(t)$ is the hazard function at time $t$ with covariates $z=\left(z_{1}, z_{2}, \ldots z_{p}\right), h_{o}(t)$ is the arbitrary baseline hazard function that characterizes how the hazard function changes as a function of survival time or the value of the hazard if all the covariates are equal to zero, $\beta=\left(\beta_{1}, \beta_{2}, \ldots \ldots \beta_{p}\right)$ is a column vector of pregression parameters associated with explanatory variables.

\subsubsection{Estimation of Parameters in Proportional Hazard Model}

Full maximum likelihood requires that we maximize with respect to the unknown parameter of interest $\beta$, and unspecified baseline hazard and survival functions. This indicates that unless we explicitly specify the baseline hazard, $h_{0}(t)$ we cannot obtain the maximum likelihood estimators for the full likelihood. But, Cox (1972) proposed using an expression he called "partial likelihood function" that depends only on the parameter of interest. Partial likelihood is a technique developed to make inference about the regression parameters in the presence of nuisance parameters $\left(h_{o}(t)\right)$ in the Cox PH model.

Thepartial likelihood function for the Cox PH model is given:

$$
L(\beta)=\prod_{j=1}^{r}\left\{\frac{\exp \left(\beta^{\prime} z_{i}\left(t_{(j)}\right)\right)}{\sum_{k \in R_{t}(j)} \exp \left(\beta^{\prime} z_{i}\left(t_{(j)}\right)\right)}\right\}
$$

Where $z_{i}\left(t_{j}\right)$ is the vector of covariate values for individual I who experience the event at $\left(t_{j}\right)$.

The general method of partial likelihood was discussed by Cox (1972).Note that this likelihood function is only for uncensored individuals. Let $t_{1}, t_{2}, \ldots \ldots \ldots \ldots, t_{n}$ be the observed survival time for $\mathrm{n}$ individuals and $\delta_{i}$ be the event indicator, which is zero if the $i^{\text {th }}$ survival time is censored and unity otherwise.

The likelihood function in equation (5) can be expressed by:

$$
L(\beta)=\left[\prod_{J=0}^{r} \frac{\exp \left(\beta^{\prime} z_{i}\left(t_{(j)}\right)\right)}{\sum_{k \in R_{t}(j)} \exp \left(\beta^{\prime} z_{i}\left(t_{(j)}\right)\right)}\right]^{\delta_{i}}
$$

Where $R\left(t_{j}\right)$ is the risk set at timet $t_{j}$. The partial likelihood is valid when there are no ties in the data set. That means are no two subjects who have the same event time.

\subsection{Accelerated Failure Time (AFT) Model}

Under AFT models we measured the direct effect of the explanatory variables on the survival time instead of hazard. This characteristic allows for an easier interpretation of the results because the parameters measure the effect of the 
correspondent covariate on the mean survival time. The members of the AFT model considered in this study are the Weibull AFT, log- logistic AFT and log-normal AFT models.

\subsubsection{Weibull Accelerated Failure Time Model}

The Weibull distribution (including the Exponential distribution as a special case) can be parameterized as an AFT model and they are the only family of distributions to have this property. The Weibull distribution is very flexible model for time-to-event data. It has a hazard rate which is monotone increasing, decreasing, or constant (Klein \&Moeschberger, 2003). The Accelerated Failure Time (AFT) representation of the survival and hazard function of the Weibull model with scale parameter and shape parameter is given by:

$$
f(t, \mu, \alpha)=\frac{\alpha}{\mu}\left(\frac{t}{\mu}\right)^{\alpha-1} \exp \left(\left(-\frac{t}{\mu}\right)^{\alpha}\right) \text {,where } \mu>0 \text { and } \alpha>0 .
$$

And the baseline hazard of this model for the $i^{\text {th }}$ subject is

$$
\mathrm{h}_{0}\left(\mathrm{t}_{\mathrm{i}}, \mathrm{x}\right)=\frac{\alpha}{\mu}\left(\frac{t}{\mu}\right)^{\alpha-1}
$$

\subsubsection{Log-logistic Accelerated Failure Time model}

The log-logistic distribution has a fairly flexible functional form, it is one of the parametric survival time models in which the hazard rate may be decreasing, increasing, as well as hump shaped that is it initially increases and then decreases. In cases where one comes across to censored data, using log-logistic distribution is mathematically more advantageous than other distributions. The log-logistic model has two parameters $\lambda$ and $\rho$ where $\lambda$ the scale parameter is and $\rho$ is the shape parameter. The probability density function of log-logistic is given as:

$$
\mathrm{f}(\mathrm{t})=\frac{\lambda \rho_{\mathrm{t}} \rho_{-1}}{\left(1+\lambda_{\mathrm{t}} \rho\right)^{2}}
$$

The corresponding survival and hazard functions are given by:

$$
\mathrm{S}(\mathrm{t})=\frac{1}{1+\lambda_{\mathrm{t}} \rho} \text { and } \mathrm{h}(\mathrm{t})=\frac{\lambda \rho_{\mathrm{t}} \rho-1}{1+\lambda_{\mathrm{t}} \rho}
$$

Where $\lambda \in R, \rho>0$

Then, the AFT representation of log-logistic survival function is given by:

$$
\mathrm{S}_{\mathrm{t}}(\mathrm{t})=\left[1+t^{\frac{1}{\sigma}} \exp \left(\frac{-\mu-\alpha \prime x}{\sigma}\right]^{-1}\right.
$$

And the associated hazard function for the $\mathrm{i}^{\text {th }}$ individual also as follow:

$$
\mathrm{h}(\mathrm{t})=\frac{1}{\sigma t}\left[1+t^{\frac{1}{\sigma}} \exp \left(\frac{-\mu-\alpha \prime x}{\sigma}\right]^{-1}\right.
$$

\subsubsection{Log-normal Accelerated Failure Time Model}

If the survival times are assumed to have a log-normal distribution, the baseline survival function and hazard function are given by (Collett, 2003):

$$
\mathrm{S}_{0}(\mathrm{t})=1-\phi\left(\frac{\log t-\mu}{\sigma}\right) \text { and } \mathrm{h}_{0}(\mathrm{t})=\frac{\phi\left(\frac{\log \mathrm{t}}{\sigma}\right)}{\left[1-\phi\left(\frac{\log t}{\sigma}\right)\right] \sigma t}
$$

Where $\mu$ and $\sigma$ are parameters, $\phi(\mathrm{x})$ is cumulative density function of the standard distribution. The survival function for the $\mathrm{i}^{\text {th }}$ individual is 


$$
\mathrm{S}_{\mathrm{i}}(\mathrm{t})=\mathrm{S}_{0}\left(\mathrm{t} / \Omega_{\mathrm{i}}\right)=\mathrm{S}_{0}\left(\mathrm{t}+\exp \left(\mu+\alpha^{\prime} \mathrm{x}_{\mathrm{i}}\right)=1-\phi\left(\frac{\log t-\alpha^{\prime} \mathrm{xi}-\mu}{\sigma}\right)\right.
$$

Where $\Omega_{\mathrm{i}}=\exp \left(\alpha_{1} x_{1}+\alpha_{2} x_{2}+\cdots+\alpha_{p} x_{p}\right)$. Therefore the $\log$ survival time for the $\mathrm{i}^{\text {th }}$ individual has normal $\left(\mu+\alpha^{\prime} \mathrm{x}_{\mathrm{i}}\right.$, $\sigma)$. The log normal distribution has the AFT property. In a two group study we can easily get $\phi^{-1}[1-\mathrm{S}(\mathrm{t})]=\frac{1}{\phi}(\log t-$ $\alpha^{\prime} x_{i}-\mu$ ), where $\mathrm{x}_{\mathrm{i}}$ is the value of a categorical variables takes the values 0 in one group and 1 in the other group.

\subsection{Shared Frailty Model}

Shared frailty model is a conditional model in which frailty is common to all subjects in a cluster. The shared frailty model is responsible for creating dependence between event times. It is also known as a mixture model because the frailties in each cluster are assumed to be random. It assumes that, the given frailty, all event times in a cluster are independent. Shared frailty model was introduced by Clayton (1978) without using the notion frailty and extensively studied by (Hougaard, Therneau \& Grambsch,2000, and Duchateau et al.,2003).It assumes that similar observations share frailty, even though frailty may vary from group to group. In effect, shared frailty causes observations within the same group to be correlated. Conditional on the random effect, called the frailty denoted by $U_{i}$, the survival times in cluster $(1 \leq \mathrm{i} \leq \mathrm{n})$ are assumed to be independent and the proportional hazard frailty model assumes:

$$
h_{i j}\left(t / x_{i j}\right)=h_{o}(t) \exp \left(\beta^{\prime} x_{i j}+u_{i j}\right)
$$

where $\mathrm{i}$ indicates the $\mathrm{i}^{\text {th }}$ cluster and $\mathrm{j}$ indicates the $\mathrm{j}^{\text {th }}$ individual for the $\mathrm{i}^{\text {th }}$ cluster, $\mathrm{h}_{\mathrm{o}}(\mathrm{t})$ is the baseline hazard function, $\mathrm{u}_{\mathrm{i}}$ the random term of all the subjects in cluster $i, X_{i j}$ the vector of covariates for subject $j$ in cluster $i$, and $\beta$ the vector of regression coefficients.

\subsubsection{Shared Gamma Frailty Distribution}

The gamma distribution is very-well known and has simple densities. It is the most common distribution used for describing frailty. Even though gamma models have closed form expressions for survival and hazard functions, from a computational view, it fits well to frailty data and it is easy to derive the closed form expressions for unconditional survival and hazard functions. To make the model identifiable, we restrict that expectation of the frailty equals one and variance be finite, so that only one parameter needs to be estimated. Thus, the distribution of frailty $\mathrm{Z}$ is the one parameter gamma distribution. Under the restriction, the corresponding density function and Laplace transformation of gamma distribution:-

$$
f_{z}\left(z_{i}\right)=\frac{z_{i}{ }^{(1 / \theta)-1} \exp \left(-\frac{z_{i}}{\theta}\right)}{\Gamma(1 / \theta) \theta^{1 / \theta}}
$$

Where $\Gamma($.$) is gamma function. It corresponds to a Gamma distribution Gam (\mu, \theta)$ with $\mu$ fixed to 1 for identifiability

and its variance is $\theta$. The associated Laplace transform is: $-L(s)=\left(1+\frac{s}{\theta}\right)^{-\theta} \quad \theta>0$

From this, if $\theta>0$, there is heterogeneity. So the large values of $\theta$ reflect a greater degree of heterogeneity among groups and a stronger association within groups.

The conditional survival function of the gamma frailty distribution (Munda et al., 2012) is given by:

$$
S_{\theta}(t)=[(1-\theta \ln \{s(t)\})]^{-1 / \theta} \quad \theta>0
$$

The conditional hazard function of the gamma frailty distribution is given by:

$$
h_{\theta}(t)=h(t)[(1-\theta \ln \{s(t)\})]^{-1}
$$

Where $\mathrm{S}(\mathrm{t})$ and $\mathrm{h}(\mathrm{t})$ are the survival and the hazard functions of the baseline distributions. Larger variance indicates a stronger association within groups. For the Gamma distribution, the Kendall's Tau (Hougaard, 2000), which measures the association between any two event times from the same cluster in the multivariate case. The associations within group members are measured by Kendall's, which is given by:

$$
\tau=\frac{\theta}{\theta+2} \tau \in(0,1)
$$




\subsubsection{Inverse Gaussian Shared Frailty Distribution}

Inverse Gaussian frailties generate stronger dependence at mid time. As an alternative to the gamma distribution, the inverse Gaussian distribution was introduced by Hougaard (1984) and has been used by (Manton et al., 1986 \& Klein et al., 1992). Similar to the gamma frailty model, simple closed-form expressions exist for the unconditional survival and hazard functions, this makes the model attractive. The probability density function of an inverse Gaussian shared distributed random variable with parameter $\theta>0$ is given by:

$$
f_{z}\left(z_{i}\right)=\left(\frac{1}{2 \pi \theta}\right)^{1 / 2} z_{i}^{-3 / 2} \exp \left(\frac{-\left(z_{i}-1\right)^{2}}{2 \theta z_{i}}\right) \quad \theta>0, \mathrm{z}>0
$$

For identifiability, we assume $\mathrm{z}$ has expected value equal to one and variance $\theta$.

The Laplace transformation of the inverse Gaussian distribution is:-

$$
L(s)=\exp \left[\frac{1-(1+2 \theta s)^{1 / 2}}{\theta}\right] \theta>0, \mathrm{~s}>0
$$

For the inverse Gaussian frailty distribution the conditional survival function is given by:

$$
s_{\theta}(t)=\exp \left\{\frac{1}{\theta}\left(1-[1-2 \theta \ln \{s(t)\}]^{1 / 2}\right)\right\} \quad \theta>0
$$

For the inverse Gaussian frailty distribution the conditional hazard function is given by:

$$
h_{\theta}(t)=h(t)[1-2 \theta \ln \{s(t)\}]^{-1 / 2} \quad \theta>0
$$

Where $\mathrm{S}(\mathrm{t})$ and $\mathrm{h}(\mathrm{t})$ are the survival and the hazard functions of the baseline distributions.

With multivariate data, an inverse Gaussian distributed frailty yields a kendall's Tau given by:

$$
\tau=\frac{1}{2}-\frac{1}{\theta}+\frac{\exp \left(\frac{2}{\theta}\right)}{\theta^{2}} \int_{\frac{2}{\theta}}^{\infty} \frac{\exp (-u)}{u} d u \in(0,0.5)
$$

\section{Result and Discussion}

\subsection{Descriptive Statistics}

The medical cards of 270 obstetric fistula patients were reviewed, 50(18.52\%) were censored at the end of the follow up and $220(81.48 \%$ ) of women physically cured. Out of which $24.81 \%$ and $50.74 \%$ were women of Age group less than 20 and 21-30 years having recurrence proportion of $20.37 \%$ and $41.48 \%$ respectively. There were $72.96 \%$ of women had weight less than or equal to $50 \mathrm{~kg}$ in which $55.56 \%$ of women physically cured. The proportion of patients who were physically cured in the marital status of married, divorced and others were $60.37 \%, 17.78 \%$ and $3.33 \%$ respectively

(Table 2).

Table 2. Distribution of recurrence of women from Obstetric fistula in south west Ethiopia.

Status

\begin{tabular}{lllll}
\cline { 3 - 4 } Covariates & Category & Censored $(\%)$ & Recurrence $(\%)$ & Total $(\%)$ \\
\hline Age & $<=20$ & $12(4.44)$ & $55(20.37)$ & $67(24.81)$ \\
& $21-30$ & $25(9.26)$ & $112(41.48)$ & $137(50.74)$ \\
& $>=30$ & $13(4.81)$ & $53(19.63)$ & $66(24.44)$ \\
\hline
\end{tabular}




\begin{tabular}{|c|c|c|c|c|}
\hline \multirow[t]{2}{*}{ Weight } & $<=50 \mathrm{~kg}$ & $47(17.41)$ & $150(55.56)$ & 197(72.96) \\
\hline & $>50 \mathrm{~kg}$ & $3(1.11)$ & $70(25.93)$ & $73(27.04)$ \\
\hline \multirow[t]{3}{*}{ Marital status } & Married & $8(2.96)$ & $163(60.37)$ & $171(63.33)$ \\
\hline & Divorced & $42(15.56)$ & $48(17.78)$ & $90(33.33)$ \\
\hline & Others & $0(0.00)$ & $9(3.33)$ & $9(3.33)$ \\
\hline \multirow[t]{3}{*}{ Parity } & 1 child & $44(16.30)$ & $39(14.44)$ & $83(30.74)$ \\
\hline & 2-4 children & $3(1.11)$ & $98(36.30)$ & 101(37.41) \\
\hline & $>=5$ children & $3(1.11)$ & $83(30.74)$ & $86(31.85)$ \\
\hline \multirow[t]{3}{*}{ Residence } & Rural & $45(16.67)$ & $214(79.26)$ & $259(95.93)$ \\
\hline & Urban & $5(1.85)$ & $6(2.22)$ & \\
\hline & & & & 11(4.07) \\
\hline \multirow[t]{2}{*}{ Education level } & Illiterate & $2(0.74)$ & $177(65.56)$ & $179(66.30)$ \\
\hline & Literate & $48(17.78)$ & $43(15.93)$ & 91(33.70) \\
\hline \multirow[t]{2}{*}{ Incontinence } & $>3$ months & $2(0.74)$ & $134(49.63)$ & $136(50.37)$ \\
\hline & $<=3$ months & $48(17.78)$ & $86(31.85)$ & $134(49.63)$ \\
\hline \multirow[t]{2}{*}{ Antenatal care visit } & No & $1(0.37)$ & 171(63.33) & $172(63.70)$ \\
\hline & Yes & $49(18.15)$ & $49(18.15)$ & $98(36.30)$ \\
\hline \multirow[t]{2}{*}{ Labor duration } & $<=2$ days & $1(0.37)$ & $82(30.37)$ & $83(30.74)$ \\
\hline & $>2$ day & $49(18.15)$ & $138(51.11)$ & $187(69.26)$ \\
\hline \multirow[t]{2}{*}{ Place of delivery } & Home & $5(1.85)$ & $174(64.44)$ & $179(66.30)$ \\
\hline & H.Institution & $45(16.67)$ & $46(17.04)$ & 91(33.70) \\
\hline
\end{tabular}




\begin{tabular}{|c|c|c|c|c|}
\hline \multirow[t]{2}{*}{ Mode of delivery } & Spontaneous V. & $(2.96)$ & 181(67.04) & 189(70.00) \\
\hline & Cesearem S. & $2(15.56)$ & $39(14.44)$ & $81(30.00)$ \\
\hline \multirow[t]{2}{*}{ Surgery approach } & Vaginal & $(2.59)$ & $186(68.89)$ & 193(71.48) \\
\hline & Abdominal & $3(15.93)$ & $34(12.59)$ & $77(28.52)$ \\
\hline \multirow[t]{2}{*}{ Duration of catheter } & $<=14$ days & $3(1.11)$ & $198(73.33)$ & $201(74.44)$ \\
\hline & 21 days & $47(17.41)$ & $22(8.15)$ & $69(25.56)$ \\
\hline \multirow[t]{2}{*}{ Status of urethra } & Completely.damaged & $6(2.22)$ & 213(78.89) & $219(81.11)$ \\
\hline & Partially damaged & $44(16.30)$ & $7(2.59)$ & $41(18.89)$ \\
\hline \multirow[t]{2}{*}{ Type of fistula } & RVF & $1(0.37)$ & $33(12.22)$ & $34(12.59)$ \\
\hline & VVF & $49(18.15)$ & 187(69.26) & $236(87.41)$ \\
\hline
\end{tabular}

Note. $\mathrm{H}=$ health, $\mathrm{V}=$ vertex, $\mathrm{S}=$ section.

\subsection{Non-parametric Survival Analysis}

4.2.1 The Kaplan- Meier Estimate of Time -to- recovery from obstetric Fistula

Non-parametric survival analysis is very important to visualize the survival of time-to-recovery of women from obstetric fistula in south west Ethiopia under different levels of the covariate. Moreover, it gives information on the shape of the survival and hazard functions of obstetric fistula data set. Survival time distributions for time-to-recovery is estimated for each group using the K-M method and in order to compare the survival curves of two or more groups, log-rank test and generalized wilcoxon test has been employed. 

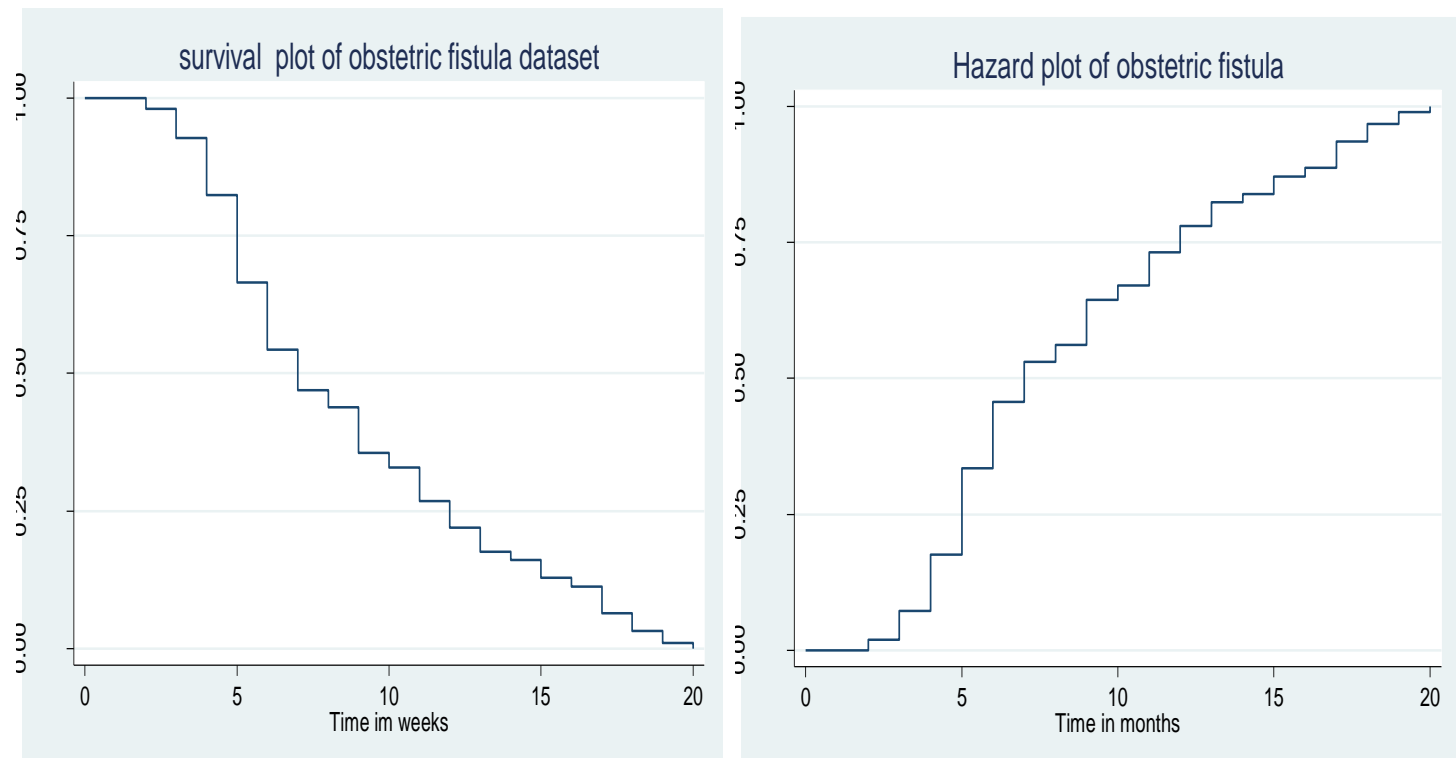

Figure 1. The K-M plots of Survival and hazard functions of obstetric fistula patients
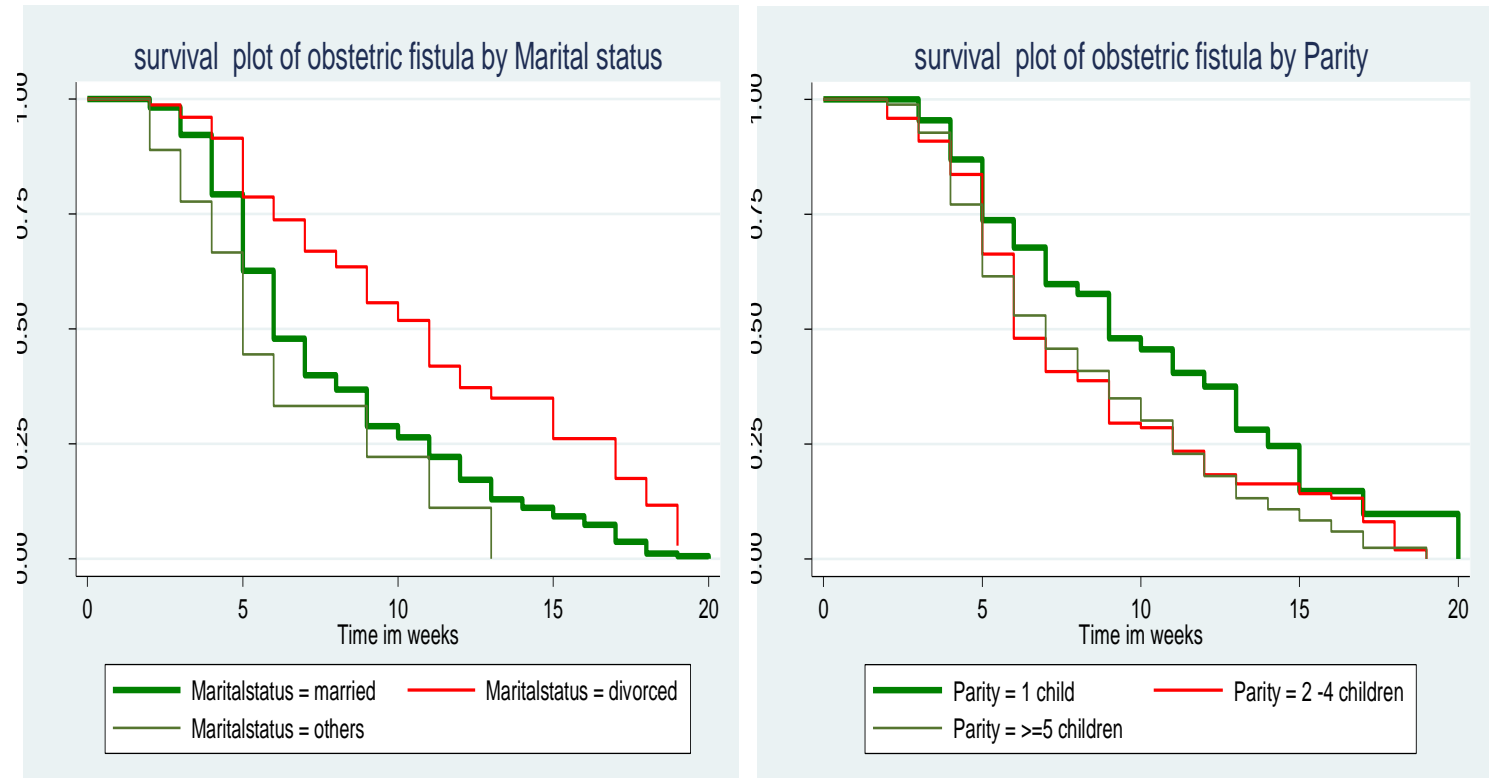

Figure 2. The Kaplan Meier plot of survival functions of Obstetric fistula by Marital status and Parity of women

\subsubsection{Comparison of Survival Experiences of Obstetric fistula}

The results of both log-rank and Breslow test for survival difference were highly significant. Log-rank showed that there is a significant difference of survival experience among groups of weight of patients, marital status, Parity, Residence, Incontinence, Antenatal care, mode of delivery, surgery approach, status of urethra and types of fistula of women. Similarly the results of generalized wilcoxon test also show that there were significant differences among obstetric fistula patients survival experience of groups weight of patients, marital status, Parity, Residence, Incontinence, Antenatal care, mode of delivery, surgery approach, status of urethra and types of fistula of women except for Parity and surgery approach which means there is no differences in survival experience of patients who had one child or 2-4 children, vaginal surgery approach and greater than 5 children and abdominal surgery approach have at the earlier phases where the number at risk is large. 
Table 3. Comparison of survival experience of Obstetric fistula using demographic, health and risk behavior variables in south west, Ethiopia

Generalized wilcoxon (Breslow)

Log-rank test

\begin{tabular}{|c|c|c|c|c|c|c|}
\hline Covariates & Chi-square & Df & Pr>chi-square & Chi-square & Df & Pr $>$ Chi-square \\
\hline Weight & 18.34 & 1 & 0.000 & 8.88 & 1 & 0.0029 \\
\hline Marital status & 19.43 & 2 & 0.0001 & 16.75 & 2 & 0.0002 \\
\hline Parity & 7.28 & 2 & 0.0263 & 5.42 & 2 & 0.0664 \\
\hline Residence & 16.74 & 1 & 0.0000 & 11.06 & 1 & 0.0009 \\
\hline Incontinence & 9.33 & 1 & 0.0023 & 9.87 & 1 & 0.0017 \\
\hline Antenatal care & 21.72 & 1 & 0.0000 & 15.68 & 1 & 0.0001 \\
\hline Mode of delivery & 9.40 & 1 & 0.0022 & 6.81 & 1 & 0.0091 \\
\hline Surgery approach & 6.29 & 1 & 0.0121 & 3.47 & 1 & 0.0625 \\
\hline Status of urethra & 20.06 & 1 & 0.0000 & 18.40 & 1 & 0.0000 \\
\hline Types of fistula & 17.57 & 1 & 0.0000 & 16.91 & 1 & 0.0000 \\
\hline
\end{tabular}

Note. Df=degree of freedom

While the Goodness of fit testing approach is employed for Cox PH model, it provides a test statistic and p-value for assessing the $\mathrm{PH}$ assumption for a given predictor of interest. The global test is less than 0.05 which indicates the PH assumptions do not satisfied by the covariate in the model. These also revealed that there is a violation of the proportional hazard assumption for the covariate Type of fistula ( $\mathrm{p}$-value=0.0174). Thus, we doubt the accuracy of the PH assumption and consider the AFT model for this data set.

\subsection{Accelerated Failure Time Model Results}

\subsubsection{Univeriate Analysis}

The univariate analysis was fitted for every covariate by Accelerated Failure Time models using different baseline distributions i.e. weibull, log-logistic, and lognormal. In all univariate analysis of AFT models, weight of women, marital status, Parity, Residence, Incontinence, Antenatal care, mode of delivery, status of urethra and types of fistula of women were significantly associated with time to recovery status at 5\% level of significance. Those variables are candidate predictors for further analysis in this study.

\subsubsection{Multivariate Accelerated Failure Time Analysis}

For time-to recovery data, Multivariate Accelerated Failure Time(AFT) model and shared frailty models of weibull, log-logistic, and log-normal distribution were fitted by including all the covariates those are significant in the univariate analysis at 5\% level of significance. To compare the efficiency of different models, the AIC and BIC were used. It is the most common applicable criterion to select model. Based on AIC and BIC, a model having the minimum AIC \&BIC values were preferred. Accordingly, Log-normal Accelerated Failure Time model (AIC = 345.0627\&BIC=391.8422) found to be the best for the time-to-recovery from obstetric fistula data set from the given alternatives when we include all the covariate those are significant in the univariate analysis. 
Table 4. Comparison of AFT models using AIC and BIC criteria for Obstetric fistula patients' data

\begin{tabular}{l|c|c}
\hline Baseline distribution & AIC & BIC \\
\hline Weibull & 359.1949 & 405.9744 \\
\hline Log-logistic & 347.5315 & 394.3110 \\
\hline Lognormal & 345.0627 & 391.8422 \\
\hline
\end{tabular}

The results lognormal multivariate AFT model showed that the predictor covariates marital status, Residence, Incontinence, Antenatal care, status of urethra and types of fistula of women were significantly associated with time to recovery status at $5 \%$ level of significance. The remaining variables which were used in the univariate covariate analysis such as weight of women, parity and mode of delivery found to be non-significant. This implies that the covariates weight, parity and mode of delivery were no jointly effect on the time to recovery of women from obstetric fistula rather individual effect on the recovery of women (Table 5). In this model, when the effect of other held constant, the estimated acceleration factor for married women is about 1.1961 with $95 \%$ confidence interval $[1.0185,1.4049]$ with p-value of 0.029. This indicates married women have prolonged survival time to physically cured from obstetric fistula than others (like single) women.

Table 5. Summary result for Log-Normal Acceleration Failure Time model

\begin{tabular}{|c|c|c|c|c|c|c|}
\hline Covariates & Categories & Estimate $(\beta)$ & $\Phi$ & $\mathrm{SE}(\beta)$ & $95 \% \mathrm{CI}$ for $\phi$ & $\mathrm{P}$-value \\
\hline Weight Ref(>50kg) & $<=50 \mathrm{~kg}$ & -0.1440 & 0.8660 & 0.0735 & {$[0.7498,1.0000]$} & 0.051 \\
\hline \multirow[t]{2}{*}{ Marital status Ref(others) } & Married & 0.1791 & 1.1961 & 0.0821 & {$[1.0185, \quad 1.4049]$} & $0.029 * *$ \\
\hline & Divorced & -0.1854 & 0.8308 & 0.1695 & {$[0.5960, \quad 1.1581]$} & 0.274 \\
\hline \multirow[t]{2}{*}{ Parity (> 5 children) } & 1 child & -0.0668 & 0.9354 & 0.0916 & {$[0.7816 \quad, 1.1193]$} & 0.466 \\
\hline & 2-4 children & -0.0832 & 0.9202 & 0.0937 & {$[0.7658,1.1056]$} & 0.375 \\
\hline Residence (urban) & Rural & 0.5314 & 1.7013 & 0.2080 & {$[1.1319, \quad 2.5574]$} & $0.011^{* *}$ \\
\hline IncotinenceRef(<=3month $)$ & $>3$ months & 0.1593 & 1.1727 & 0.0682 & $1.0260,1.3403]$ & $0.019 * *$ \\
\hline Antenatal care Ref(yes) & No & 0.1811 & 1.1985 & 0.0792 & {$[1.0262, \quad 1.3996]$} & $0.022 * *$ \\
\hline Mode of delivery $\operatorname{Ref}(\mathrm{CS})$ & Spontaneous & 0.0936 & 1.0981 & 0.0853 & {$[0.9289, \quad 1.2980]$} & 0.273 \\
\hline Status of urethra & Completely & 0.4299 & 1.5371 & 0.1635 & {$[1.1157,2.1176]$} & $0.009 * *$ \\
\hline Ref(partially D) & D. & & & & & \\
\hline Type of fistula Ref(VVF) & RVF & -0.2928 & 0.7462 & 0.1022 & {$[0.6107, \quad 0.9116]$} & $0.004 * *$ \\
\hline
\end{tabular}

Scale $\sigma=0.4914$ and shape $\alpha=2.035, \Phi$ Indicates Acceleration factor; $* *$ significant at $5 \%$ level; $95 \%$ CI: $95 \%$ confidence interval foracceleration factor; $\operatorname{SE}(\beta)$ : standard error for $\beta$; Ref. Reference, $D=$ Damaged 


\subsection{Parametric Shared Frailty Model Results}

In the previous section (section 4.3), three AFT models were fitted and compared to analyze the survival of time-to-recovery from obstetric fistula to identify baseline distribution and associated risk factors. To model the heterogeneity (random component) using zone as frailty term and investigate risk factors associated with the survival of time-to-recovery from obstetric fistula gamma shared frailty and inverse Gaussian shared frailty model with log normal baseline distribution were used. The effect of random component (frailty) was significant for log-normal gamma shared frailty models, but insignificant for inverse Gaussian shared frailty models and lognormal gamma shared frailty model have minimum akaike information criteria (AIC =344.77) event smaller AIC of lognormal AFT models. This indicates log-normal gamma shared frailty model is more efficient model to describe time-to-recovery from obstetric fistula dataset.

The frailty term in this model is assumed to follow a gamma distribution with mean 1 and variance equal to theta $(\theta)$. The estimated value of theta $(\theta)$ is 0.86 . A likelihood ratio test for the hypothesis $\theta=0$ is shown in the (Table 6) below and indicates a chi-square value of 73.57 with one degree of freedom resulted a highly significant P-value of 0.000 . This implied that the frailty component had significant contribution to the model. And the associated Kendall's tau ( $\tau$ ), which measures dependence within clusters (Zone), is estimated to be 0.30 .

The confidence intervals of the acceleration factor for all significant categorical covariates do not include one at 5\% level of significance. This showed that they are significant factors for determining the survival of time-to-recovery among women in Jimma south west Ethiopian. The acceleration factor for women who are lived in rural area was 1.7591 with $95 \%$ CI $[1.1737,2.6363]$ times greater than those who are lived in urban area $(P-v a l u e=0.011)$, this indicates rural women have prolonged time-to-recovery than urban women. In addition, the acceleration factor and its $95 \%$ confidence interval of Incontinence for women was 1.1727 and $(1.0260,1.3403)$ respectively. The confidence interval did not include one and p-value was very small $(\mathrm{P}=0.006)$ indicating that incontinence status of women was significant factor to determine the survival time of time-to-recovery from fistula at $5 \%$ level of significance. This showed that the timing of physically cured for incontinence greater than three months women was longer than incontinence less than or equal to three months women. Similarly, women who hadn't Antenatal care, status of urethra with completely damaged and married women have prolonged survival time to physically cured than women who had Antenatal care, status of urethra with partially damaged and others women.

Table 6. Results of final log- normal gamma shared frailty model of obstetric fistula patients.

\begin{tabular}{|c|c|c|c|c|c|c|}
\hline Covariate & Category & Estimate $(\beta)$ & $\Phi$ & $\operatorname{SE}(\beta)$ & $95 \% \mathrm{CI}$ for $\phi$ & P-value \\
\hline \multirow[t]{3}{*}{ Marital status } & Ref(others) & & & & & \\
\hline & Married & 0.1862 & 1.2047 & 0.0800 & {$[1.0298, \quad 1.4092]$} & $0.020^{*}$ \\
\hline & Divorced & -0.1911 & 0.8260 & 0.1708 & {$[0.5911,1.1544]$} & 0.263 \\
\hline \multirow[t]{2}{*}{ Residence } & Ref(urban) & & & & & \\
\hline & Rural & 0.5648 & 1.7591 & 0.2064 & {$[1.1737, \quad 2.6363]$} & $0.006^{* *}$ \\
\hline \multirow[t]{2}{*}{ Incontinence } & $\operatorname{Ref}(<=3$ months $)$ & & & & & \\
\hline & $>3$ months & 0.1638 & 1.1780 & 0.0680 & {$[1.0311,1.3458]$} & $0.016^{* *}$ \\
\hline \multirow[t]{2}{*}{ Antenatal care } & Ref(yes) & & & & & \\
\hline & No & 0.1978 & 1.2187 & 0.0791 & {$[1.0436, \quad 1.4230]$} & $0.012^{* *}$ \\
\hline \multirow[t]{2}{*}{ Status of urethra } & Ref(partially D.) & & & & & \\
\hline & Completely D. & 0.4668 & 1.5949 & 0.1576 & {$[1.1710, \quad 2.1723]$} & $0.003 * *$ \\
\hline
\end{tabular}




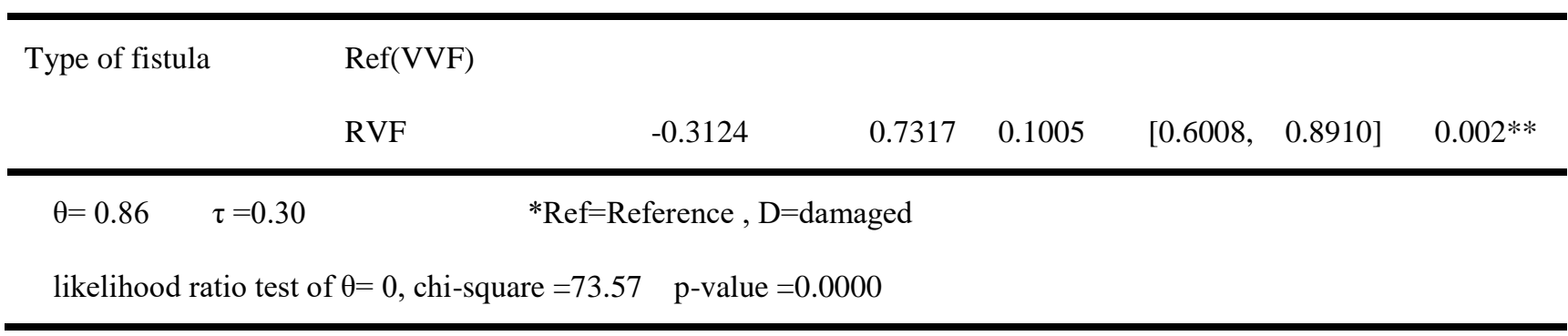

\subsection{Discussions}

The findings of this study revealed that there is heterogeneity between patients categorized as Zone and correlation within the same Zone of obstetric fistula patients. The Results obtained were found to be analogous with literature on the topic. Based on the given dataset marital status of the women was the first factors that affect survival time of recovery from Obstetric fistula. As it was indicated in both log-normal Accelerated failure time model and log-normal gamma shared frailty models the Acceleration factor of married women with obstetric fistula in south west Ethiopia is about 1.1961and 1.2047 times prolonged survival time-to-recovery after physically treated than women who were others (like single) marital status respectively.Residence of a patient is another, prognostic factor that significantly predicts the recovery time of patients from obstetric fistula. The lognormal acceleration factor for women who lived in Rural area is much higher $(\phi$ $=1.7013$ ). That is, rural patients more likely prolonged time to recover than urban women. The final lognormal gamma shared frailty model also indicated living being in rural prolonged time to physically cured of women. The result is comparable with earlier study [Johnson K, 2007 \& Vangeen derhuysen, 2001].

The result of this study suggested that Antenatal care of women was significant predictive factor for time- to- recovery of women from obstetric fistula. Both lognormal Accelerated failure time and gamma shared frailty models showed that having Antenatal care shorten time to recovery. Women who hadn't Antenatal care have acceleration factor 1.1727 and 1.2187 respectively. That is, the survival time of women to recovery were prolonged by $17.27 \%$ and $21.87 \%$ higher than women who had Antenatal care.

Duration of incontinence of women having obstetric fistula is a potential risk factors that significantly predicts survival of time to recovery of women from obstetric fistula. The multiple covariate analysis of Accelerated failure time showed that, Acceleration factor of having incontinence greater than three month is $17.27 \%$ prolonged than women who had incontinence less than or equal to three months and lognormal gamma shared frailty models also indicates that acceleration factor is much higher (longer time) for women those of having incontinence greater than three months $(\phi$ $=1.1780$ ).

Status of urethra is an important predictor of time to recovery of women. Both lognormal Accelerated failure time and gamma shared frailty models suggest that the Status of urethra significantly associated with women's recovery from obstetric fistula. Women who had completely damaged status of urethra have acceleration factor of 1.5371 which is prolonged the time of recovery than women who had partially damaged status of urethra or having partially damaged urethra need shorten time to physically cure. This is similar to reports by (Hussen \& Melese, 2017) in Harar city, who reported partially urethral damaged having Associate hazard ratio $(\mathrm{AHR}=0.6929)$ and complete bladder neck distraction $(\mathrm{AHR}=0.1324)$ have significant effect on mean time to recovery from obstetric fistula.

Another potential risk factor that accelerates recovery status of women from vaginal fistula is types of fistula. Results of lognormal accelerated failure time and gamma shared frailty model showed that the types of fistula have significantly associated with survival time of recovery from obstetric fistula.

\section{Conclusions}

To model the determinants of time-to-recovery, different parametric shared frailty and AFT models by using different baseline distributions were applied. Among this using AIC, log-normal gamma shared frailty model is better fitted to time-to-recovery from obstetric fistula dataset than other parametric shared frailty and AFT models. There was a frailty (clustering) effect on the time-to-recovery from obstetric fistula that arises due to differences in distribution of timing of recovery among zones in south west of Ethiopia. This indicates the presence of heterogeneity and necessitates the frailty models.

The result of Log-normal AFT and Log-normal-gamma shared frailty models showed that marital status, place of residence, incontinence, Antenatal care, Status of urethra and types of fistula of women were found significant predictors to time-to-recovery of women among women with obstetric fistula in south west Ethiopia. Among these significant predictors, marital status, place of residence, duration of incontinence, Antenatal care and status of urethra of women with 
obstetric fistula prolong timing of physically cure (recovery) while having Recto-Vaginal fistula(RVF) types of fistula of women shorten timing of recovery for women with obstetric fistula. It recommended that awareness have to be given for the society on those risk factors and the future studies also need to assess the level of awareness, treatment and control of these risk factors.

\section{References}

Clayton, D. (1978). Model for Association in Bivariate Life Tables and Its Application in Epidemiological Studies of Familial Tendency in Chronic Disease Incidence. Biometrika. 65, 141-151, https://doi.org/10.1093/biomet/65.1.141

Collett, D. (2003). Modelling Survival Data in Medical Research (2nd edition). Chapman and Hall, London.

Cook, R. J., Dickens, B. M., \& Syed, S. (2004). Obstetric fistula: The challenge to human rights.International Journal of Gynecology \& Obstetrics, 87(1), 72-77. https://doi.org/10.1016/j.ijgo.2004.07.005.

Cox, D. (1972). Regression models and life tables (with discussions). Journal of the Royal Statistical Society. 34, 187-220.

Duchateau, L., Janssen, P., Kezic, I., \& Fortpied, C. (2003). Evolution of recurrent asthma event rate over time in frailty models. Journal of the Royal Statistical Society (B) 52, $355-363$.

Duchateau, L., \& Janssen, P. (2008). The Frailty Model. Springer: New York.

Goodwin, W. E., \& Scardino, P. T. (1980). 'Vesicovaginal and Ureterovaginal Fistulas: A Summary of 25 Years of Experience', J Urol, 123, 370-374.

Holme, A., Breen, M., \& MacArthur, C. (2007). Obstetric fistulae: A study of women managed at the monze mission hospital, zambia. BJOG: An International Journal of Obstetrics \&Gynaecology, 114(8), 1010-1017. https://doi.org/10.1111/j.1471-0528.2007.01353.x.

Hougaard, P. (2000). Analysis Of Multivariate Survival Data. Springer, New York, 1, 255-273.

Hougaard, P. (1984). Life table methods for heterogeneous populations. Biometrika, 71,75-83.

Hussen, S., \& Melese, E. (2017). Time-to-recovery from obstetric fistula and associated factors: The case of Harar Hamlin Fistula Center.The Ethiopian Journal of Health Development (EJHD), 31(2).

Johnson, K. (2007). Incontinence in Malawi: analysis of a proxy measure of vaginal fistula in a national survey.Int $J$ Gynaecol Obstet., 99(Suppl 1):S122-9.https://doi.org/10.1016/j.ijgo.2007.06.033.

Kaplan\& Meier. (1958).Non-parametric estimation from incomplete observations.Journal of American Statistical Association.

Kazaura, M. R., Kamazima, R. S., \&Mangi, E. J. (2011). Perceived causes of obstetric fistulae from rural southern Tanzania. African Health Sciences, 11(3), 377-382.

Kirby, A, Gleason, J, Greer, W., Norman, A., Lengmang, S., \& Richter, H. (2014). Characterization of colorectal symptoms in women with vesicovaginal fistulas. International Journal of obstetrics and Gynaecology, 116(1), 64-66.

Klein, J. (1992). Survival analysis: techniques for censored and truncated data. Medical College of Wisconsin.

Langkilde, N. C., Pless, T. K., Lundbeck, F., \& Nerstrom, B. (1999). 'Surgical Repair of Vesicovaginal Fistulae: A Ten-Year Retrospective Study.', Scand J Urol Nephrol, 33(2), 100-103.

Manton, K., Stallard, E., \& Vaupel, J. (1986). Alternative models for heterogeneity of mortality risks among the aged. Journal of the American Statistical Association., 81, 635-644.

Mselle, L., Moland, K., Evjen-Olsen, B., Mvungi, A., \& Kohi, T. (2011). "I am nothing": experiences of loss among women suffering from severe birth injuries in Tanzania. Biomedical Central Women's Health., 11, 49.

Muleta, M., Hamlin, C.', Fantahun, M., Kennedy, R. C., \& Tafesse, B. (2008). 'Health and Social Problems Encountered by Treated and Untreated Obstetric Fistula Patients in Rural Ethiopia. ', International Journal of Obstetrics and Gynaecology. 112, 1328-1330.

Munda, M., Rotolo, F., \& Legrand, C. (2012). Parametric Frailty Models in R. Journal of American Statistical Association, 55, 1-21.

Therneau, T. M., \& Grambsch, P. M. (2000). Modeling Survival Data. New York: Springer.

Tunçalp, Ö., Tripathi, V., Landry, E., \& et al. (2015). Measuring the incidence and prevalence ofobstetric fistula: approaches, needs and recommendations. Bull World HealthOrgan, 93, 60-62. 
Vangeenderhuysen, C., Prual, A., \& Ould, D. (2001). Obstetric fistulae: incidence estimates for sub-Saharan Africa.Int J Gynaecol Obs., 73, 65-66.

Wall, L. L., Arrowsmith, S. D., Briggs, N. D., \& et al. (2005). The Obstetric Vesicovaginal Fistula inthe Developing World. Obstetrics \& Gynecology Survey. 60(7), S3-S47.

\section{Copyrights}

Copyright for this article is retained by the author(s), with first publication rights granted to the journal.

This is an open-access article distributed under the terms and conditions of the Creative Commons Attribution license (http://creativecommons.org/licenses/by/4.0/). 